\title{
SN 2011dh: DISCOVERY OF A TYPE IIb SUPERNOVA FROM A COMPACT PROGENITOR IN THE NEARBY GALAXY M51
}

\author{
Iair Arcavi ${ }^{1}$, Avishay Gal-Yam $^{1}$, Ofer Yaron $^{1}$, Assaf Sternberg $^{1}$, Itay Rabinak $^{1}$, Eli Waxman $^{1}$, \\ Mansi M. Kasliwal ${ }^{2,21}$, Robert M. QuimbY ${ }^{2,21}$, Eran O. OfeK ${ }^{2,21}$, Assaf Horesh ${ }^{2,21}$, Shrinivas R. KulkarNi ${ }^{2,21}$, \\ Alexei V. Filippenko ${ }^{3}$, Jeffrey M. Silverman ${ }^{3}$, S. Bradley Cenko ${ }^{3}$, Weidong Li ${ }^{3}$, Joshua S. Bloom ${ }^{3}$, Mark Sullivan ${ }^{4}$, \\ Peter E. Nugent ${ }^{3,5,21}$, Dovi Poznanski ${ }^{3,5,21}$, Evgeny Gorbikov ${ }^{6}$, Benjamin J. Fulton ${ }^{7}$, D. Andrew Howell ${ }^{7,8}$, \\ David Bersier $^{9}$, Amedee Riou ${ }^{10}$, Stephane Lamotte-Bailey ${ }^{11}$, Thomas Griga ${ }^{12}$, Judith G. Cohen ${ }^{2}$, Stephan Hachinger ${ }^{13}$, \\ David Polishook ${ }^{1}$, Dong Xu ${ }^{1}$, Sagi Ben-Ami ${ }^{1}$, Ilan Manulis ${ }^{1}$, Emma S. Walker ${ }^{14}$, Kate Maguire ${ }^{4}$, Yen-Chen Pan ${ }^{4}$, \\ Thomas Matheson ${ }^{15}$, Paolo A. Mazzali ${ }^{13,16}$, Elena Pian ${ }^{14,17}$, Derek B. Fox ${ }^{18}$, Neil Gehrels ${ }^{19}$, Nicholas Law ${ }^{20}$, \\ Philip James ${ }^{9}$, Jonathan M. Marchant ${ }^{9}$, Robert J. Smith ${ }^{9}$, Chris J. Mottram ${ }^{9}$, Robert M. Barnsley ${ }^{9}$, \\ Michael T. KANDRASHOFF ${ }^{3}$, AND Kelsey I. ClubB ${ }^{3}$ \\ ${ }^{1}$ Department of Particle Physics and Astrophysics, The Weizmann Institute of Science, Rehovot 76100, Israel; iair.arcavi@weizmann.ac.il \\ ${ }^{2}$ Cahill Center for Astrophysics, California Institute of Technology, Pasadena, CA 91125, USA \\ ${ }^{3}$ Department of Astronomy, University of California, Berkeley, CA 94720-3411, USA \\ ${ }^{4}$ Department of Physics (Astrophysics), University of Oxford, Keble Road, Oxford, OX1 3RH, UK \\ ${ }^{5}$ Computational Cosmology Center, Lawrence Berkeley National Laboratory, 1 Cyclotron Road, Berkeley, CA 94720, USA \\ ${ }^{6}$ The Wise Observatory and the Raymond and Beverly Sackler School of Physics and Astronomy, the Faculty of Exact Sciences, \\ Tel Aviv University, Tel Aviv 69978, Israel \\ ${ }^{7}$ Las Cumbres Observatory Global Telescope Network, 6740 Cortona Dr, Suite 102, Goleta, CA 93117, USA \\ ${ }^{8}$ Physics Department, University of California, Santa Barbara, Broida Hall, Mail Code 9530, Santa Barbara, CA 93106-9530, USA \\ ${ }^{9}$ Astrophysics Research Institute, Liverpool John Moores University, Twelve Quays House, Egerton Wharf, Birkenhead CH41 1LD, UK \\ ${ }_{10}$ Asnieres 49370, Becon les Granits, France \\ ${ }^{11} 14$ rue de Dammarie, 77000 Melun, France \\ 12 Am Derkmannsstueck 34, Schwerte, D-58239, Germany \\ ${ }^{13}$ Max Planck Institute for Astrophysics, Garching, Germany \\ ${ }^{14}$ Scuola Normale Superiore di Pisa, Pisa 56126, Italy \\ ${ }^{15}$ National Optical Astronomy Observatory, NOAO System Science Center, 950 North Cherry Avenue, Tucson, AZ 85719, USA \\ ${ }^{16}$ INAF, Osservatorio Astronomico di Padova, Italy \\ ${ }^{17}$ INAF, Astronomical Observatory of Trieste, Via G.B. Tiepolo 11, I-34143 Trieste, Italy \\ ${ }^{18}$ Astronomy and Astrophysics, Eberly College of Science, The Pennsylvania State University, University Park, PA 16802, USA \\ ${ }^{19}$ Astrophysics Science Division, NASA Goddard Space Flight Center, Greenbelt, MD 20771, USA \\ ${ }^{20}$ Dunlap Institute for Astronomy and Astrophysics, University of Toronto, 50 St. George Street, Ontario, Toronto M5S 3H4, Canada \\ Received 2011 June 19; accepted 2011 October 4; published 2011 November 4
}

\begin{abstract}
On 2011 May 31 UT a supernova (SN) exploded in the nearby galaxy M51 (the Whirlpool Galaxy). We discovered this event using small telescopes equipped with CCD cameras and also detected it with the Palomar Transient Factory survey, rapidly confirming it to be a Type II SN. Here, we present multi-color ultraviolet through infrared photometry which is used to calculate the bolometric luminosity and a series of spectra. Our early-time observations indicate that SN 2011dh resulted from the explosion of a relatively compact progenitor star. Rapid shock-breakout cooling leads to relatively low temperatures in early-time spectra, compared to explosions of red supergiant stars, as well as a rapid early light curve decline. Optical spectra of SN 2011dh are dominated by H lines out to day 10 after explosion, after which He I lines develop. This SN is likely a member of the cIIb (compact IIb) class, with progenitor radius larger than that of SN 2008ax and smaller than the eIIb (extended IIb) SN 1993J progenitor. Our data imply that the object identified in pre-explosion Hubble Space Telescope images at the SN location is possibly a companion to the progenitor or a blended source, and not the progenitor star itself, as its radius $\left(\sim 10^{13} \mathrm{~cm}\right)$ would be highly inconsistent with constraints from our post-explosion spectra.
\end{abstract}

Key words: supernovae: individual (PTF11eon/SN2011dh)

Online-only material: color figures, machine-readable table

\section{INTRODUCTION}

At the end of their lives, massive stars $\left(M \gtrsim 8 M_{\odot}\right)$ explode as core-collapse supernovae ( $\mathrm{SNe}$ ), leaving a neutron star or a black hole remnant. Several different types of $\mathrm{SNe}$ have been observed, including H-rich Type II-P/II-L SNe, H-rich interacting Type IIn SNe, H-poor Type IIb SNe, H-less Type Ib SNe, and H- and He-less Type Ic SNe (see Filippenko 1997 for a review).

\footnotetext{
${ }^{21}$ Einstein Fellow.
}

Direct progenitor detections in pre-explosion images have established a link between low-mass red supergiants (RSGs) and SNe IIP, the most common type of core-collapse SN (see Smartt 2009 for a review). Evidence also exists linking $\mathrm{SNe}$ IIn with much more massive progenitors (Gal-Yam et al. 2007; Gal-Yam \& Leonard 2009; Smith et al. 2011b). However, the mapping between massive star classes and types of $\mathrm{SNe}$ is far from complete, due to both theoretical gaps and to the scarcity of observational constraints. Numerous core-collapse $\mathrm{SNe}$ are discovered each year and statistical 


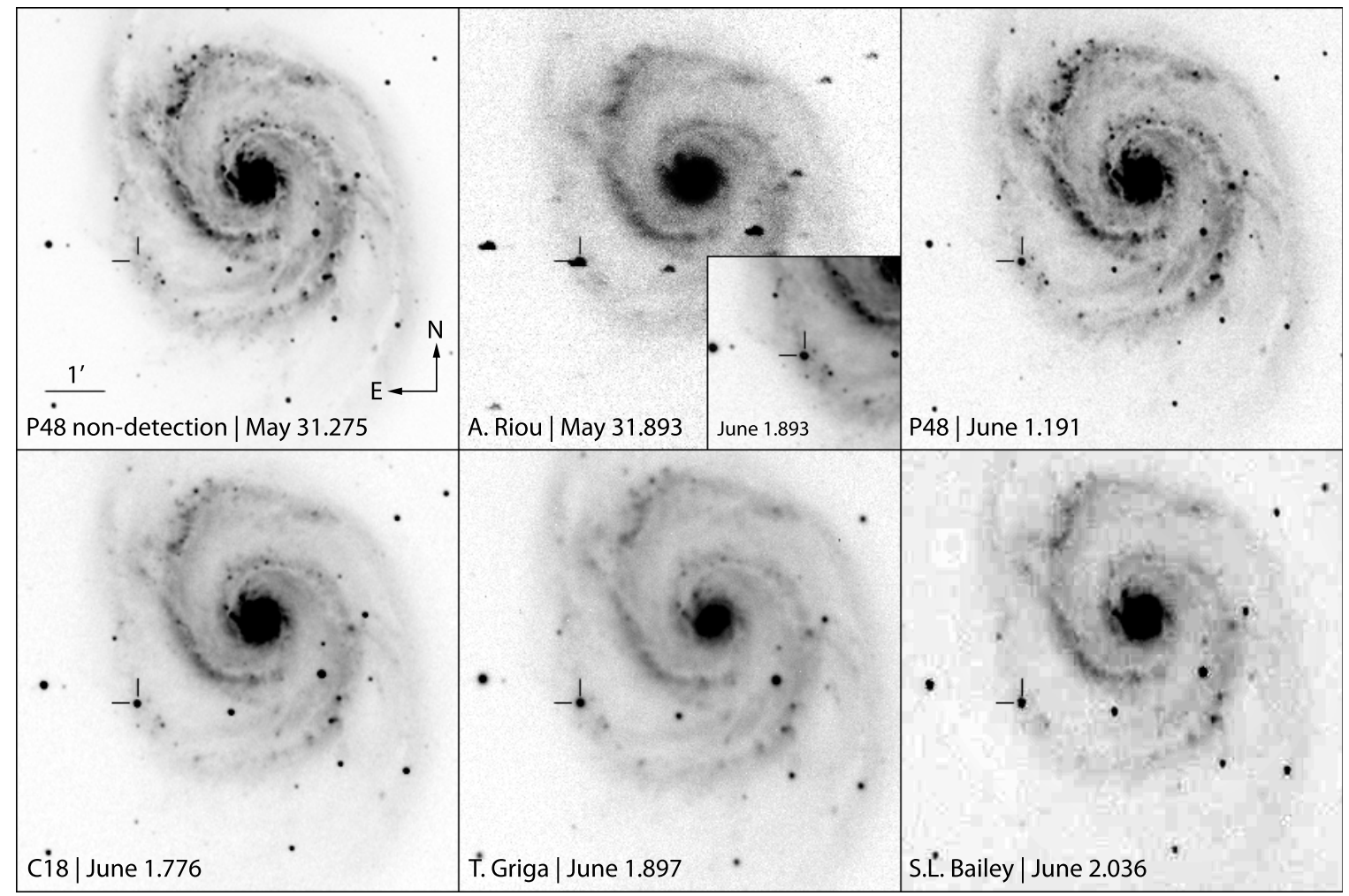

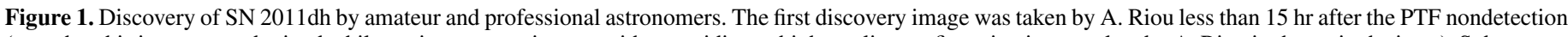

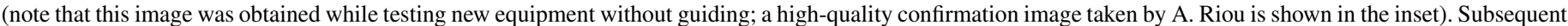
discovery and confirmation images are also shown. UT dates are used throughout.

analyses of large samples are yielding interesting insights (e.g., Anderson \& James 2009; Boissier \& Prantzos 2009; Arcavi et al. 2010; Li et al. 2011; Smith et al. 2011a), but some of the most robust information comes from studying the few nearest events.

The Palomar Transient Factory (PTF; Rau et al. 2009; Law et al. 2009) is a wide-field variability survey. Our short-cadence strategy and real-time capability (Gal-Yam et al. 2011) enables the discovery of SNe shortly after the explosion. Some parts of the sky, such as those with photogenic nearby galaxies, are observed by amateur astronomers even more frequently. Thus, the amateur community may be the first to discover and report bright events in these nearby hosts, as is the case here.

We report the discovery of SN 2011dh (Silverman et al. 2011; also named PTF11eon), a Type IIb SN in M51, a nearby interacting spiral galaxy located at a distance of $8.03 \pm 0.77 \mathrm{Mpc}$ (retrieved from the NASA/IPAC Extragalactic Database, $\mathrm{NED}^{22}$ ). We find rapid light curve evolution at early times, as well as relatively cool early spectra compared with those of PTF10vdl (Gal-Yam et al. 2011) and SN 1993J (Wheeler et al. 1993). Our data suggest that SN 2011dh resulted from a progenitor more compact than that of the prototypical Type IIb SN 1993J (Filippenko et al. 1993), but perhaps more extended than the progenitor of the Type IIb SN 2008ax (Pastorello et al. 2008; Chornock et al. 2011).

\section{DISCOVERY}

The SN was discovered in images taken on May 31.893 (UT times are used throughout this paper) by A. Riou in

\footnotetext{
22 NED, http://nedwww.ipac.caltech.edu/
}

France $^{23}$ (Figure 1). Approximately $7 \mathrm{hr}$ later, on 2011 June 1.191 , the PTF detected the SN in images taken by the Palomar 48 in Oschin Schmidt Telescope (P48). The SN was seen at $\alpha(\mathrm{J} 2000)=13^{\mathrm{h}} 30^{\mathrm{m}} 05^{\mathrm{s}} .08$ and $\delta(\mathrm{J} 2000)=+47^{\circ} 10^{\prime} 11^{\prime \prime} .2$ (astrometry good to $0^{\prime \prime}$. 1 , based on the Sloan Digital Sky Survey, SDSS ${ }^{24}$ ) at a magnitude of $m_{g}=13.15 \pm 0.09$, and it was absent from a PTF image taken by the P48 on 2011 May 31.275 down to a $3 \sigma$ limiting magnitude of $m_{g}=21.44$. Subsequent imaging was undertaken at the Wise Observatory 18 in telescope on June 1.776, by T. Griga on June 1.897 and by S. L. Bailey on June 2.036. Data collected by amateur astronomers during the first hours of this event are very helpful in determining the explosion time. The data described above constrain the explosion time to be between May 31.275 and May 31.893; however, this time window can still be decreased (A. Gal-Yam et al. 2011, in preparation). SN 2011dh is the third SN to be discovered in M51 in the last 17 years, after the subluminous Type IIP SN 2005cs (e.g., Li et al. 2006b) and the Type Ic SN 1994I (e.g., Filippenko et al. 1995).

Li \& Filippenko (2011a, 2011b) identify a star at the approximate location of the SN in pre-explosion Hubble Space Telescope (HST) images. Maund et al. (2011) confirm this with adaptive optics to within 23 mas and Van Dyk et al. (2011) improve the location accuracy to 7 mas. Maund et al. (2011) further claim that this star, an F8 supergiant with $\log \left(L / L_{\odot}\right)=4.92 \pm 0.20$ and $T_{\text {eff }}=6000 \pm 280 \mathrm{~K}$ (which implies a radius of $\sim 10^{13} \mathrm{~cm}$ ), is the progenitor of SN 2011dh. Here we show that this is unlikely.

\footnotetext{
23 CBAT "Transient Objects Confirmation Page" entry PSN J13303600+4706330.

${ }^{24}$ SDSS, http://www.sdss.org
} 
Table 1

Photometric Observations of SN 2011dh

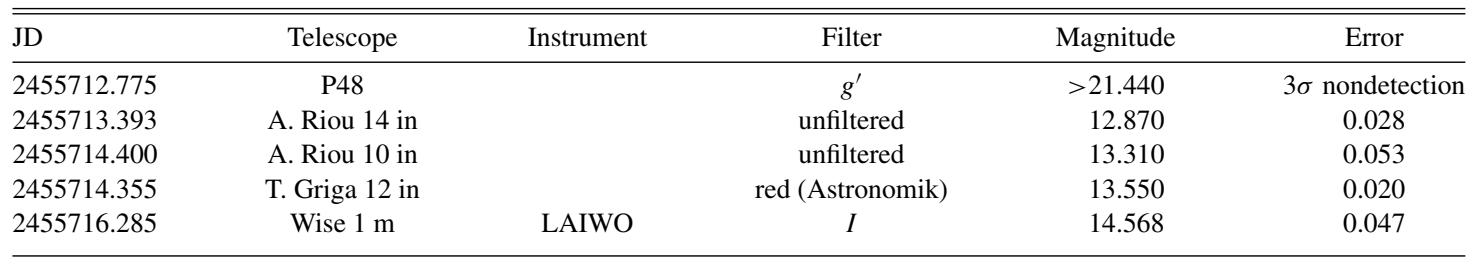

(This table is available in its entirety in a machine-readable form in the online journal. A portion is shown here for guidance regarding its form and content.)

\section{FOLLOW-UP OBSERVATIONS}

\subsection{Photometry}

Immediately after discovery, we initiated an extensive followup campaign. Optical and infrared (IR) photometry was obtained at P48, the Wise Observatory $1 \mathrm{~m}$ and 18 in (C18) telescopes, the Byrne Observatory at Sedgwick Reserve (BOS) $0.8 \mathrm{~m}$ telescope, and the Peters Automated InfraRed TELescope (PAIRITEL). Our photometry is performed on difference images by constructing a reference frame from data taken before the SN explosion and then subtracting that from each frame in which the SN is present, matching the point-spread functions (PSFs) of the two images (Gal-Yam et al. 2004; Gal-Yam et al. 2008). We calibrate the Wise data and BOS $B$-band data using Landolt (1992) standards. The BOS $i^{\prime}$-band data were calibrated to SDSS. We use the average magnitudes obtained from the same filter and instrument taken consecutively on the same night. For the P48 data, the SN photometry was measured using a PSF fitting method after image subtraction, due to saturation of the SN in the images. ${ }^{25}$ In each image frame, the PSF was determined from nearby field stars, and this average PSF was then fit at the position of the SN, weighting each pixel according to Poisson statistics. Saturated pixels (when present) have their weight set to zero. P48 images where the SN was brighter than $12.5 \mathrm{mag}$ were not used. We calibrate our P48 light curve to the SDSS system using SDSS observations of the same field, and including both color and color-airmass terms to determine the zero point of each image (the root-mean square of these color-term fits is $\sim 0.015$ mag with respect to published SDSS magnitudes). For the PAIRITEL data, all individual frames from the same night were stacked using the Swarp ${ }^{26}$ software. Reference frames for the subtractions were taken from the Two Micron All Sky Survey $(2 \mathrm{MASS})^{27}$ using the HOTPANTS ${ }^{28}$ software. The subtracted images were then calibrated with respect to point sources from 2MASS. Calibration and subtraction errors were summed in quadrature.

The Ultraviolet/Optical Telescope (UVOT) on board Swift followed SN 2011dh, and preliminary results from these observations were reported by Kasliwal \& Ofek (2011) and Arcavi et al. (2011). We retrieved the level-2 UVOT data for SN $2011 \mathrm{dh}$ from the Swift data archive. To increase the signal-to-noise ratio, we stacked the images for each individual filter on a daily basis. To remove host-galaxy contamination underlying the SN location, we subtracted pre-outburst images of M51 obtained by the UVOT. We caution that, due to the nonlinearity of the coincidence-loss correction, such a technique can lead to mod-

\footnotetext{
25 From day 9 and onward.

$26 \mathrm{http} / / / \mathrm{www}$.astromatic.net/software/swarp

27 http://www.ipac.caltech.edu/2mass

$28 \mathrm{http} / / /$ www.astro.washington.edu/users/becker/hotpants.html
}

est systematic uncertainties in the SN flux, particularly at high count rates. For the $U$ filter, we employed the photometric calibration described by Li et al. (2006a) to measure the photometry of SN 2011dh in the subtracted images. For the three UV filters (UVW1, UVW2, and UVM2), the calibration from Poole et al. (2008) was adopted. Many tests have shown that the two calibrations by Li et al. (2006a) and Poole et al. (2008) are consistent with each other for bright sources.

We note that the images by A. Riou and T. Griga were taken using nonstandard photometric filters or no filters at all. We calibrate them to the $R$ filter, and thus expect some systematic error due to filter differences. Because reference images are not available for these data, we perform aperture photometry on the SN with no image subtraction.

Our photometry is corrected for Galactic extinction using the Schlegel et al. (1998) maps via NED and applying the Cardelli et al. (1989) algorithm assuming $R_{V}=3.1$. We do not correct for host-galaxy extinction, as it is found to be small (see below). We adopt a distance modulus of $29.52 \mathrm{mag}$ and present our photometry in the Vega system (except for the P48, $i^{\prime}$-band BOS, and UVOT data which are calibrated to the AB system).

Our photometric data are presented in the top panel of Figure 2 and in Table 1. The initial decline in the light curve on days $1-3.5$, prior to the rise to peak magnitude, can be interpreted as a detection of the shock-breakout cooling tail (e.g., Chevalier 1992; Waxman et al. 2007; Chevalier \& Fransson 2008; Nakar $\&$ Sari 2010). Such photometric behavior is reminiscent of that seen in the Type IIb SN 1993J (Richmond et al. 1994), but SN $2011 \mathrm{dh}$ exhibits a more rapid decline.

We also calculate a bolometric light curve (bottom panel of Figure 2) using the Swift, Wise, BOS, and PAIRITEL data between days 6 and 13 after explosion. We find that the flux contained in the $J, H$, and $K$ filters is roughly constant during this period $(33.78 \% \pm 0.85 \%)$, and that the flux beyond $2 \mu \mathrm{m}$ is negligible. We assume a constant IR flux for the interval 14-37 day after explosion, where no PAIRITEL data are available, and we also plot the possible error due to a larger $(40 \%)$ or smaller $(0 \%)$ IR flux at these times.

\subsection{Spectroscopy}

Our first spectrum was obtained with the Low Resolution Imaging Spectrometer (LRIS) mounted on the Keck-I $10 \mathrm{~m}$ telescope on June 3.304 (2.4 day after the SN discovery), identifying the event as an SN II (Silverman et al. 2011). Additional spectra were later obtained with the Kast spectrograph on the Lick Observatory $3 \mathrm{~m}$ Shane telescope, the DOLORES spectrograph on the TNG $3.6 \mathrm{~m}$ telescope, the RC spectrograph on the KPNO $4 \mathrm{~m}$ Mayall telescope, the ISIS spectrograph on the William Herschel $4.2 \mathrm{~m}$ telescope, and the FRODOspec spectrograph on the Liverpool $2 \mathrm{~m}$ Telescope (LT). All spectral frames were wavelength calibrated using standard lamp spectra. Flux 

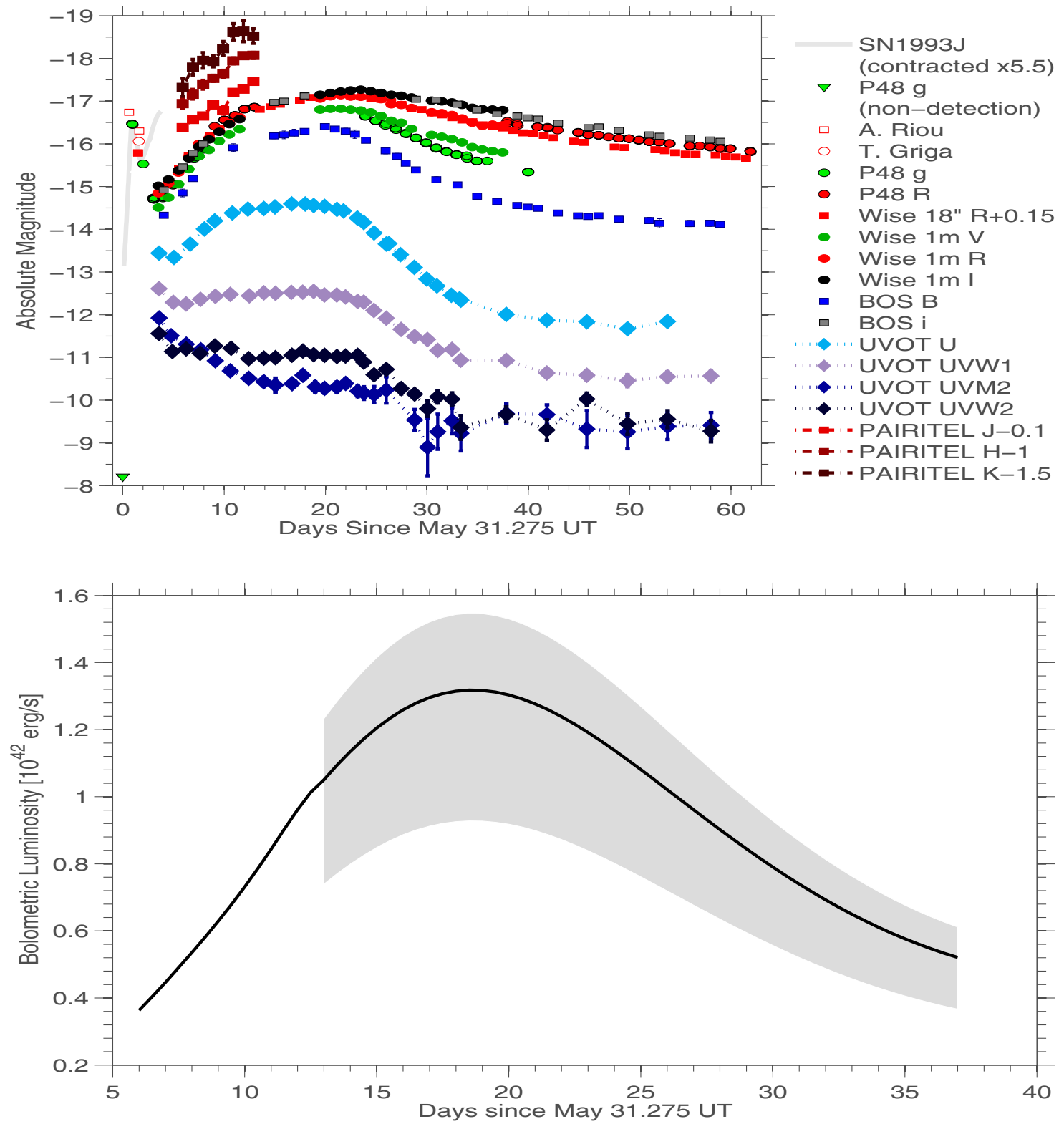

Figure 2. Top panel: the multi-band light curve of SN 2011dh. For comparison, the early-time light curve of SN 1993J from Richmond et al. (1994) is shown in gray, contracted in time by a factor of 5.5, and fainter by $0.65 \mathrm{mag}$. It is obvious that the evolution of SN 2011dh at early times is much faster than that of SN 1993J, and it is also possibly a little fainter (although uncertainties in distance determination to the events should be kept in mind). Where the error bars are not visible, they are smaller than the symbol sizes. Bottom panel: the bolometric luminosity of SN 2011dh, based on the Swift, Wise, BOS, and PAIRITEL data for days 6-13, and on the Swift, Wise, and BOS data for days 14-37, assuming a constant fraction of IR flux consistent with the average flux measured from available PAIRITEL data. The shaded area represents the effect of changing the IR flux fraction between $0 \%$ and $40 \%$.

(A color version of this figure is available in the online journal.)

calibrations were applied using a solution derived from standard stars. All of the reductions were carried out using IRAF and IDL routines. The spectra are available through the WIS Experimental Astrophysics Spectroscopy System. ${ }^{29}$

In order to estimate the extinction in the vicinity of the SN, we obtained two high-resolution spectra using the High Resolution Echelle Spectrometer (HIRES) mounted on the Keck-I $10 \mathrm{~m}$ telescope. The spectral resolution was $R=\lambda / \Delta \lambda=36,000$ $(\Delta \lambda=0.164 \AA$ in the vicinity of the Na I D $\lambda \lambda 5890,5896 \AA$ lines). The data were reduced using the MAuna Kea Echelle Extraction (MAKEE) pipeline, written by T. Barlow. ${ }^{30}$ The

\footnotetext{
${ }^{29} \mathrm{http}: / /$ www.weizmann.ac.il/astrophysics/wiseass

$30 \mathrm{http}: / /$ spider.ipac.caltech.edu/staff/tab/makee/
}

spectra were flux normalized and the equivalent width of the Na I D absorption feature was measured using the IRAF "splot" tool.

The total equivalent widths of the $\mathrm{NaI} \mathrm{D}_{2}$ and $\mathrm{D}_{1}$ lines, as measured from the HIRES spectra, were found to be $188 \pm 8 \mathrm{~m} \AA$ and $107 \pm 6 \mathrm{~m} \AA$, respectively. Using the correlation presented by Munari \& Zwitter (1997) for the $\mathrm{Na} \mathrm{I}_{1} \mathrm{D}_{1}$ line, we constrain the local extinction value to $E(B-V)<0.05$ mag. Dust reddening is therefore negligible, and we ignore it in our analysis.

\section{ANALYSIS}

We used SYNOW (Jeffery \& Branch 1990) to identify features in the spectra of SN 2011dh and find prominent $\mathrm{H}$ features as well as $\mathrm{Ca}$ II and Fe-peak elements characteristic of 


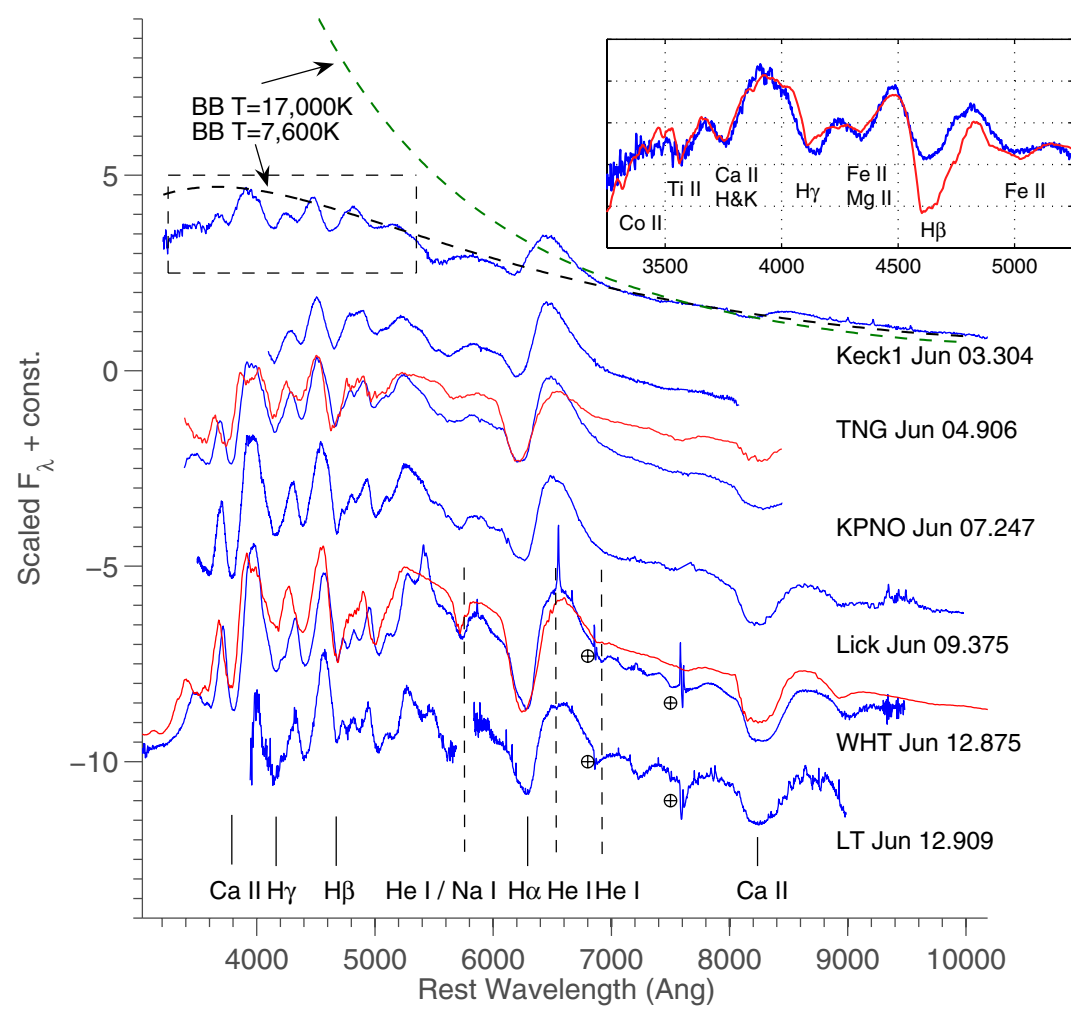

Figure 3. Spectra of SN 2011dh. The first spectrum, taken $\sim 2.4$ days after explosion, shows highly developed low-ionization features (SYNOW fit in red shown in inset) and a blackbody temperature of $\sim 7600 \mathrm{~K}$ (dashed black line). It is highly inconsistent with a blackbody temperature of 17,000 K (dashed green line) implied by a progenitor star with radius $10^{13} \mathrm{~cm}$. Radiative transfer models (red) of the spectra on June 7 and 12 indicate $\mathrm{H}$ and $\mathrm{He}$ masses of $0.024 M_{\odot}$ and $0.016 M_{\odot}$, respectively, at $v>8300 \mathrm{~km} \mathrm{~s}^{-1}$. The last two spectra, taken on the same night, show He I lines emerging (the $6678 \AA$ line is obscured by the narrow $\mathrm{H} \alpha$ feature from the underlying host galaxy in the WHT spectrum, but is present in the LT IFU spectrum which shows much less host contamination). The H lines are plotted at $13,000 \mathrm{~km} \mathrm{~s}^{-1}$, the He I lines at $6500 \mathrm{~km} \mathrm{~s}^{-1}$, and the Ca II lines at 12,000 $\mathrm{km} \mathrm{s}^{-1}$. Telluric absorption features are marked.

(A color version of this figure is available in the online journal.)

SNe II (Figure 3). We see these features at velocities as high as $\sim 17,000 \mathrm{~km} \mathrm{~s}^{-1}$ initially and at an earlier stage compared to other young SNe II, which tend to show a blue continuum with low-contrast $\mathrm{H} \alpha$ emission a few days after explosion (Figure 4). Unlike SN 1993J, no prominent He features are seen in the spectra of SN 2011dh, even out to day 10 after explosion. However, hints of He absorption appear on June 12 (Figure 3) and strong He lines develop in later spectra (Marion et al. 2011). We have further modeled two spectra using the radiative transfer code of Mazzali (2000) with a non-LTE treatment of $\mathrm{H}$ and $\mathrm{He}$ (Hachinger 2011). On June 12, the ejecta are optically thin outside $8300 \mathrm{~km} \mathrm{~s}^{-1}$. In this zone, we find $0.024 M_{\odot}$ of $\mathrm{H}$ and $0.016 M_{\odot}$ of $\mathrm{He}$ (much more He can presumably be found below $8300 \mathrm{~km} \mathrm{~s}^{-1}$ ).

Focusing on our earliest spectrum, the low continuum temperature ( $7600 \mathrm{~K}$ from a blackbody fit; Figure 3 ) and low-ionization elements indicate rapid cooling after the shock breakout. These are notably different from early-time spectra of SNe IIP (e.g., PTF10vdl; Gal-Yam et al. 2011) known to arise from RSG stars or from early-time spectra of SN 1993J (e.g., Wheeler et al. 1993; Figure 4) whose progenitor size was $\sim 4 \times 10^{13} \mathrm{~cm}$ (Woosley et al. 1994). Indeed, using Equation (13) from Rabinak \& Waxman (2011) with a radius of $10^{13} \mathrm{~cm}$, we find that two days after explosion the color temperature should be greater than 17,000 K (as observed for RSG explosions such as PTF10vdl; Gal-Yam et al. 2011). ${ }^{31}$

\footnotetext{
31 We note that the effective temperature, which is $\sim 20 \%$ lower than the color temperature, is still higher than the recombination temperature of $\mathrm{H}$, indicating that the assumption of ionized ejecta made by the model is consistent.
}

It is highly unlikely that the radius of the progenitor of SN $2011 \mathrm{dh}$ was of order $10^{13} \mathrm{~cm}$, as claimed by Maund et al. (2011) and Prieto \& Hornoch (2011), although the latter authors do emphasize the need for a smaller progenitor than that of SN 1993J. Therefore, the object identified in pre-explosion HST images (Maund et al. 2011; Van Dyk et al. 2011) is probably not the single progenitor of SN 2011dh. Rather, the progenitor could be in a binary system or a member of a compact cluster. A relatively faint Wolf-Rayet star could have a radius as small as $10^{11} \mathrm{~cm}$, while possibly remaining below the magnitude limits imposed by the $H S T$ data (Van Dyk et al. 2011).

Chevalier \& Soderberg (2010) estimate a radius of $\sim 10^{11} \mathrm{~cm}$ for the progenitor of SN 2008ax, which shows similar spectral properties to those of SN 2011dh (Figure 4). Pastorello et al. (2008) constrain the length of the shock-breakout cooling phase of SN 2008ax to be less than a few hours, implying that the radius of the progenitor of SN 2011dh could be larger than that of SN 2008ax (yet still much smaller than that of SN 1993J). We leave detailed modeling of the shock-breakout cooling phase to a future report.

We conclude that SN 2011dh is likely an SN IIb from a compact progenitor (termed cIlb by Chevalier \& Soderberg 2010).

\section{SUMMARY}

We have presented our discovery of SN 2011dh, an SN in M51, as well as results from our early follow-up effort. This event displays similarities to the Type IIb SNe 1993J and 2008ax, but also important differences. The early-time evolution 


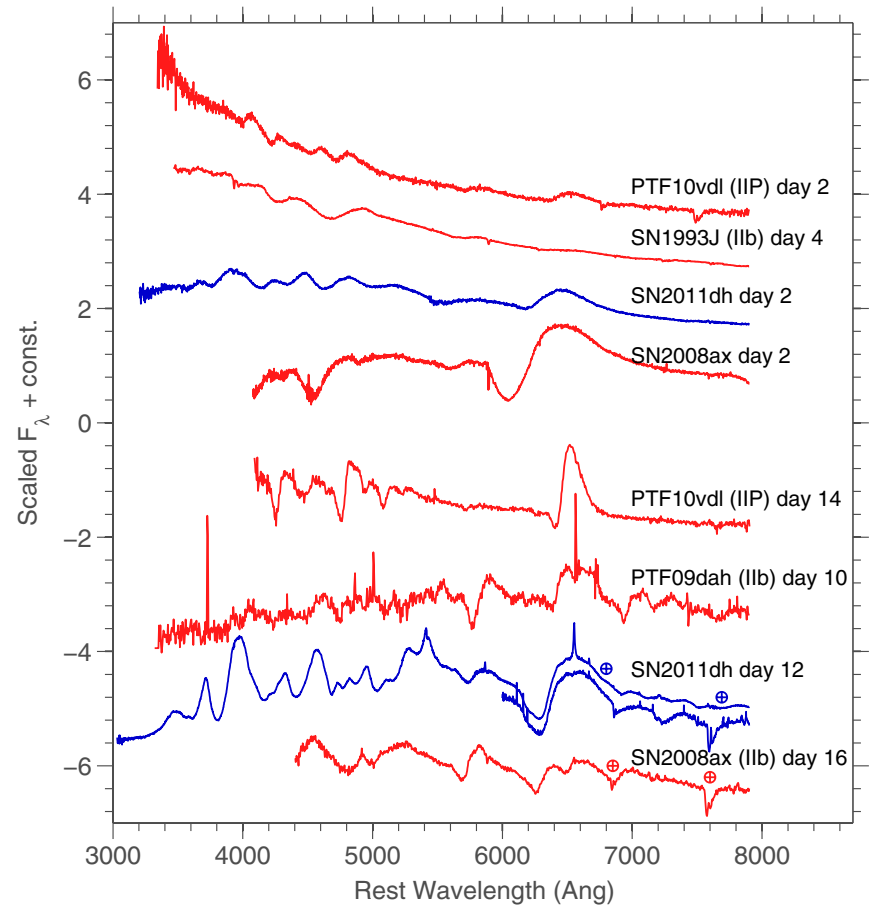

Figure 4. Comparison of the spectra of SN 2011dh at roughly 2 and 12 days with those of PTF10vdl (SN IIP; Gal-Yam et al. 2011), SN 1993J (SN eIIb; spectrum obtained by the Asiago SN Group and retrieved via SUSPECT), SN 2008ax (SN cIIb; early spectrum from Chornock et al. 2011, late spectrum from Pastorello et al. 2008), and PTF09dah (SN IIb almost identical to SN 1993J; I. Arcavi et al. 2011, in preparation). In the early-time spectra (top) SN 2011dh is not as blue as PTF10vdl and SN 1993J, and shows more developed features (including neutral $\mathrm{H}$ lines) compared to these $\mathrm{SNe}$, as expected from a smaller progenitor. SN 2008ax shows similar properties, but at higher velocities, consistent with an even smaller radius. At later times (bottom) SN 2011dh does not yet show prominent He features characteristic of Type IIb events such as PTF11dah; however, these lines developed a few days later (Marion et al. 2011).

(A color version of this figure is available in the online journal.)

of the light curve is very rapid compared to that of SN 1993J. The relatively low temperature implied by our first spectrum indicates that SN 2011dh resulted from the explosion of a fairly compact star, which is not consistent with the object identified by Maund et al. (2011) as a single star. Rather, as suggested by Van Dyk et al. (2011), the progenitor system is possibly composed of a binary system of which the progenitor of SN $2011 \mathrm{dh}$ is a member.

SN 2011dh demonstrates the importance of real-time detections and of rapid and continuous follow-up observations as crucial tools for constraining both explosion and progenitor properties via shock-breakout analysis (Soderberg et al. 2008; Ofek et al. 2010). Obtaining and analyzing a statistical sample of such young $\mathrm{SNe}$ will serve to probe the progenitor stars of a much larger sample, beyond the $\sim 20 \mathrm{Mpc}$ limit for direct progenitor detections, placing strong and novel constraints on the last phases of massive stellar evolution.

The Weizmann Institute PTF partnership is supported by the Israeli Science Foundation via grants to A.G. Collaborative work between A.G. and S.R.K. is supported by the US-Israel Binational Science Foundation. A.G. further acknowledges support from the EU FP7 Marie Curie program via an IRG fellowship and a Minerva grant. P.E.N. is supported by the US Department of Energy Scientific Discovery through Advanced Computing program under contract DE-FG02-06ER06-04. M.S. acknowledges support from the Royal Society; M.S. and A.G. are also grateful for a Weizmann-UK Making Connections grant. A.V.F.'s supernova group at U.C. Berkeley acknowledges generous support from Gary and Cynthia Bengier, the Richard and Rhoda Goldman Fund, US National Science Foundation grant AST-0908886, and the TABASGO Foundation. J.S.B. acknowledges support of an NSF-CDI grant 0941742 and NSF/AAG grant NSF/AST-100991. P.M., E.P., and E.S.W. acknowledge financial support from INAF through PRIN INAF 2009.

Instrumentation at Wise Observatory was funded in part by the Israel Space Agency (ISA), the Max Planck Institute for Astronomy (MPA) in Heidelberg, Germany, the German Israeli Science Foundation for Research and Development, and the Israel Science Foundation. The WHT is operated by the Isaac Newton Group in the Spanish Observatorio del Roque de los Muchachos of the Instituto de Astrofisica de Canarias. The Byrne Observatory at Sedgwick (BOS) is operated by the Las Cumbres Observatory Global Telescope Network. The W. M. Keck Observatory is operated as a scientific partnership among the California Institute of Technology, the University of California, and NASA; it was made possible by the generous financial support of the W. M. Keck Foundation. PAIRITEL is operated by the Smithsonian Astrophysical Observatory (SAO) and supported by the Harvard University Milton Fund, the University of Virginia, SAO, UC Berkeley, and NASA via Swift Guest Investigator programs NNX09AQ66Q and NNX10A128G. We are grateful to the dedicated staffs at all of the observatories where we obtained data.

The National Energy Research Scientific Computing Center, which is supported by the Office of Science of the U.S. Department of Energy under Contract No. DE-AC02-05CH11231, provided staff, computational resources, and data storage for this project.

\section{REFERENCES}

Anderson, J. P., \& James, P. A. 2009, MNRAS, 399, 559

Arcavi, I., Gal-Yam, A., Kasliwal, M. M., et al. 2010, ApJ, 721, 777

Arcavi, I., Gal-Yam, A., Polishook, D., et al. 2011, ATel, 3413, 1

Boissier, S., \& Prantzos, N. 2009, A\&A, 503, 137

Cardelli, J. A., Clayton, G. C., \& Mathis, J. S. 1989, ApJ, 345, 245

Chevalier, R. A. 1992, ApJ, 394, 599

Chevalier, R. A., \& Fransson, C. 2008, ApJ, 683, L135

Chevalier, R. A., \& Soderberg, A. M. 2010, ApJ, 711, L40

Chornock, R., Filippenko, A. V., Li, W., et al. 2011, ApJ, 739, 41

Filippenko, A. V. 1997, ARA\&A, 35, 309

Filippenko, A. V., Barth, A. J., Matheson, T., et al. 1995, ApJ, 450, L11

Filippenko, A. V., Matheson, T., \& Ho, L. C. 1993, ApJ, 415, L103

Gal-Yam, A., Kasliwal, M. M., Arcavi, I., et al. 2011, ApJ, 736, 159

Gal-Yam, A., \& Leonard, D. C. 2009, Nature, 458, 865

Gal-Yam, A., Leonard, D. C., Fox, D. B., et al. 2007, ApJ, 656, 372

Gal-Yam, A., Maoz, D., Guhathakurta, P., \& Filippenko, A. V. 2008, ApJ, 680, 550

Gal-Yam, A., Moon, D.-S., Fox, D. B., et al. 2004, ApJ, 609, L59

Hachinger, S. 2011, PhD thesis, Techische Univ. München

Jeffery, D. J., \& Branch, D. 1990, in Supernovae, Jerusalem Winter School for Theoretical Physics, Vol. 6, ed. J. C. Wheeler, T. Piran, \& S. Weinberg (Singapore: World Scientific), 149

Kasliwal, M. M., \& Ofek, E. O. 2011, ATel, 3402, 1

Landolt, A. U. 1992, AJ, 104, 340

Law, N. M., Kulkarni, S. R., Dekany, R. G., et al. 2009, PASP, 121, 1395

Li, W., \& Filippenko, A. V. 2011a, ATel, 3399, 1

Li, W., \& Filippenko, A. V. 2011b, ATel, 3401, 1

Li, W., Jha, S., Filippenko, A. V., et al. 2006a, PASP, 118, 37

Li, W., Leaman, J., Chornock, R., et al. 2011, MNRAS, 412, 1441

Li, W., Van Dyk, S. D., Filippenko, A. V., et al. 2006b, ApJ, 641, 1060

Marion, G. H., Kirshner, R., Wheeler, J. C., et al. 2011, ATel, 3435,

Maund, J. R., Fraser, M., Ergon, M., et al. 2011, ApJ, 739, L37

Mazzali, P. A. 2000, A\&A, 363, 705 
Munari, U., \& Zwitter, T. 1997, A\&A, 318, 269

Nakar, E., \& Sari, R. 2010, ApJ, 725, 904

Ofek, E. O., Rabinak, I., Neill, J. D., et al. 2010, ApJ, 724, 1396

Pastorello, A., Kasliwal, M. M., Crockett, R. M., et al. 2008, MNRAS, 389, 955

Poole, T. S., Breeveld, A. A., Page, M. J., et al. 2008, MNRAS, 383, 627

Prieto, J. L., \& Hornoch, K. 2011, ATel, 3433, 1

Rabinak, I., \& Waxman, E. 2011, ApJ, 728, 63

Richmond, M. W., Treffers, R. R., Filippenko, A. V., et al. 1994, AJ, 107, 1022

Rau, A., Kulkarni, S. R., Law, N. M., et al. 2009, PASP, 121, 1334

Schlegel, D. J., Finkbeiner, D. P., \& Davis, M. 1998, ApJ, 500, 525
Silverman, J. M., Filippenko, A. V., \& Cenko, S. B. 2011, ATel, 3398, 1

Smartt, S. J. 2009, ARA\&A, 47, 63

Smith, N., Li, W., Filippenko, A. V., \& Chornock, R. 2011a, MNRAS, 412, 1522

Smith, N., Li, W., Miller, A. A., et al. 2011b, ApJ, 732, 63

Soderberg, A. M., Berger, E., Page, K. L., et al. 2008, Nature, 453, 469

Van Dyk, S. D., Li, W., Cenko, S. B., et al. 2011, arXiv:1106.2897

Waxman, E., Mészáros, P., \& Campana, S. 2007, ApJ, 667, 351

Wheeler, J. C., Barker, E., Benjamin, R., et al. 1993, ApJ, 417, L71

Woosley, S. E., Eastman, R. G., Weaver, T. A., \& Pinto, P. A. 1994, ApJ, 429, 300 